\title{
INFLUÊNCIA DA RAZÃO MOLAR COMBUSTÍVEL/OXIDANTE NA MICROESTRUTURA DE ÓXIDO DE ZINCO OBTIDO POR SÍNTESE DE COMBUSTÃO EM SOLUÇÃO
}

\author{
Waleska Campos Guaglianoni ${ }^{*}$, Ana Paula Garcial, Tania Maria Basegio ${ }^{1}$, Carlos Pérez Bergmann ${ }^{1}$ \\ ${ }^{1}$ Departamento de Materiais (PPGE3M), Escola de Engenharia, Universidade Federal do Rio Grande do Sul, 90035190, Rio Grande \\ do Sul, Brasil.
}

*E-mail: waleskaguaglianoni@yahoo.com.br

\section{RESUMO}

Óxido de zinco $(\mathrm{ZnO})$ nanoestruturado foi obtido por síntese de combustão em solução (SCS) utilizando nitrato de zinco como oxidante e ureia como combustível. O material foi caracterizado por difração de raios X (DRX); método Branauer, Emmet e Teller (BET); análises termogravimétricas (ATG) e termo diferencial (ATD) e microscopia eletrônica de varredura (MEV). A influência de diferentes razões molares combustível/oxidante na microestrutura do $\mathrm{ZnO}$ foi investigada. As amostras com maior razão molar apresentaram menor tamanho de cristalito e maior área superficial. As imagens de MEV mostraram placas irregulares e placas finas com pontas alongadas que se assemelham a flores.

Palavras-chave: Óxido de zinco nanoestruturado. Síntese de combustão em solução. Microestrutura.

\section{Introdução}

$\mathrm{O}$ óxido de zinco ( $\mathrm{ZnO})$ é um semicondutor não estequiométrico do tipo $n$, Possui alta energia de ligação (60meV), bandgap de aproximadamente $3,37 \mathrm{eV}$, elevada atividade óptica e luminescente [1]. Além de ser considerado um excelente material para a área dos semicondutores, o $\mathrm{ZnO}$ tem aplicações em células solares [2], sensores de gases [3], fotocatálise [4], varistores [5], dispositivos piezoelétricos [6], indústria farmacêutica e de cosméticos [7].

As propriedades químicas e microestruturais do $\mathrm{ZnO}$ dependem do método de obtenção utilizado. $\mathrm{O} \mathrm{ZnO}$ pode ser obtido através de diversas rotas processuais: o método sol-gel, a síntese hidrotermal [8], o método Pechini [9], a combustão induzida por microondas [10], e a síntese de combustão em solução [11-13]. Dentre as rotas processuais empregadas, a síntese de combustão em solução (SCS) destaca-se por ser uma técnica simples, econômica e rápida; que produz pós finos, homogêneos e geralmente cristalinos [14]. A base da SCS procede dos conceitos termodinâmicos utilizados no campo de explosivos e propelentes [15]. As formulações são definidas a partir da composição estequiométrica da mistura redox, calculada através do balanço de valências das espécies presentes na mistura. A composição estequiométrica corresponde à reação de máxima energia liberada [11].

A SCS possui um mecanismo de reação bastante complexo, com um grande número de variáveis que afetam os produtos de síntese obtidos, dentre elas: temperatura de chama, composição química dos precursores, razão combustível/oxidante e volume de gases gerados na reação [16]. Neste sentido, o presente trabalho visa investigar a síntese por combustão em solução do $\mathrm{ZnO}$ utilizando ureia e nitrato de zinco como precursores e avaliar a influência da razão combustível/oxidante nas características microestruturais dos produtos obtidos.

\section{Parte Experimental}

Neste trabalho utilizou-se a síntese por combustão em solução para obtenção do $\mathrm{ZnO}$. As soluções precursoras foram preparadas utilizando nitrato de zinco hexahidratado $\left(\mathrm{Zn}\left(\mathrm{NO}_{3}\right) .6 \mathrm{H}_{2} \mathrm{O}\right)$ como oxidante e ureia $\left(\mathrm{CO}\left(\mathrm{NH}_{2}\right)_{2}\right)$ como combustível. As razões combustível/oxidante foram definidas de acordo com modelamento termodinâmico baseado na química dos propelentes e explosivos [15], e foram calculadas em relação à equação estequiométrica. A Tabela 1 apresenta a quantidade de combustível utilizado nas reações estudadas e sua respectiva razão molar combustível/oxidante.

Tabela 1 - Quantidade de combustível utilizada nas reações e respectiva razão molar combustível/oxidante.

\begin{tabular}{ccc}
\hline Amostra & $\begin{array}{c}\text { Quantidade } \\
\text { combustível }(\%)\end{array}$ & $\begin{array}{c}\text { Razão molar } \\
\text { combustível/oxidante }\end{array}$ \\
\hline $\mathrm{U} 1$ & -50 & 0,4 \\
\hline $\mathrm{U} 2$ & -25 & 0,6 \\
\hline $\mathrm{U} 3$ & Estequiométrica & 0,8 \\
\hline $\mathrm{U} 4$ & +25 & 1,0 \\
\hline $\mathrm{U} 5$ & +50 & 1,2 \\
\hline
\end{tabular}


A Figura 1 apresenta o fluxograma do procedimento experimental adotado para a síntese de $\mathrm{ZnO}$. O nitrato de zinco foi dissolvido em aproximadamente $10 \mathrm{~mL}$ de água deionizada. A solução foi aquecida a $60^{\circ} \mathrm{C}$ sob agitação constante. A ureia foi adicionada após 10 minutos. Para garantir a homogeneização da solução, manteve-se a agitação por mais 10 minutos. Elevou-se a temperatura de operação da chapa elétrica para $500^{\circ} \mathrm{C}$. Os produtos da reação de síntese foram calcinados em forno elétrico tipo mufla (marca SANCHIS) na temperatura de $600^{\circ} \mathrm{C}$, com uma taxa de aquecimento de $2{ }^{\circ} \mathrm{C} / \mathrm{min}$ e tempo de patamar de 3 horas. Após o tratamento térmico, os pós-obtidos foram caracterizados por difração de raios X (DRX), método Branauer, Emmet e Teller (BET), análise termogravimétrica (ATG) e termo diferencial (ATD), e microscopia eletrônica de varredura (MEV).

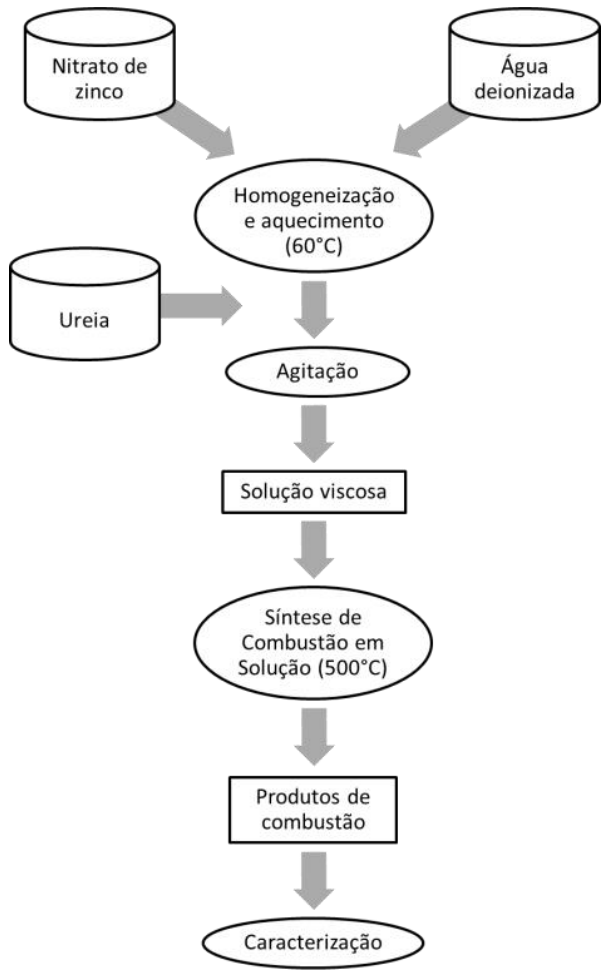

Figura 1 - Fluxograma do procedimento experimental da síntese de $\mathrm{ZnO}$.

As fases cristalográficas e os tamanhos de cristalitos foram determinados utilizando $\mathrm{o}$ difratômetro de raios $\mathrm{X}$ PHILIPS (modelo X'Pert MPD), equipado com um monocromador de grafite, radiação $\mathrm{Cu}-\mathrm{K} \alpha(\lambda=1,5406)$ e ânodo fixo operado a $40 \mathrm{kV}$ e $40 \mathrm{~mA}$. O tamanho de cristalito foi calculado com o software WinFitt. Este software realiza um ajuste nos picos experimentais utilizando funções assimétricas Pearson VII, corrige o alargamento instrumental e decompõe os picos em duas componentes: Gaussiana e Lorentziana. A contribuição Gaussiana caracteriza o efeito no tamanho dos cristalitos e a contribuição Lorentziana caracteriza o efeito da deformação na largura dos picos de difração. Assim, o tamanho dos cristalitos pode ser calculado, determinando as funções Pearson VII específicas para cada máximo de difração [17].

A área superficial dos pós-obtidos foi determinada pelo modelo proposto por Branauer, Emmett e Teller a partir de isotermas de adsorção [18], utilizando o equipamento Autosorb Quantachrome (modelo Nova 1000). As isotermas de adsorção são calculadas a partir de informações referentes à adsorção do gás nitrogênio $\left(\mathrm{N}_{2}\right)$ na superfície do material analisado. O equipamento calcula a área superficial total da amostra através do volume de nitrogênio adsorvido em função da pressão relativa (P/P0). Para obter a área superficial específica, a área superficial medida é dividida pelo peso total da amostra analisada [19].

As análises termogravimétricas (ATG) e termo diferencial (ATD) foram realizadas com um analisador térmico Mettler (modelo TGA SDTA 851E) a uma taxa de aquecimento de $20^{\circ} \mathrm{C} / \mathrm{min}$ e uma vazão de $40 \mathrm{~L} / \mathrm{min}$ de oxigênio.

A morfologia dos produtos da reação foi observada com um microscópio eletrônico de varredura (MEV, JEOL-JSM 6060).

\section{Resultados e discussões}

A Figura 2 mostra os difratogramas de raios $\mathrm{X}$ dos produtos da síntese de $\mathrm{ZnO}$ por combustão em solução. Os picos característicos do óxido de zinco (JCPDS 01-089-0510) são observados em todas as amostras. Os pós-obtidos com menor razão molar combustível/oxidante apresentaram picos de difração de raios $\mathrm{X}$ mais intensos, sugerindo que as amostras são mais cristalinas. Todas as formulações mostraram suficiente grau de cristalinidade.

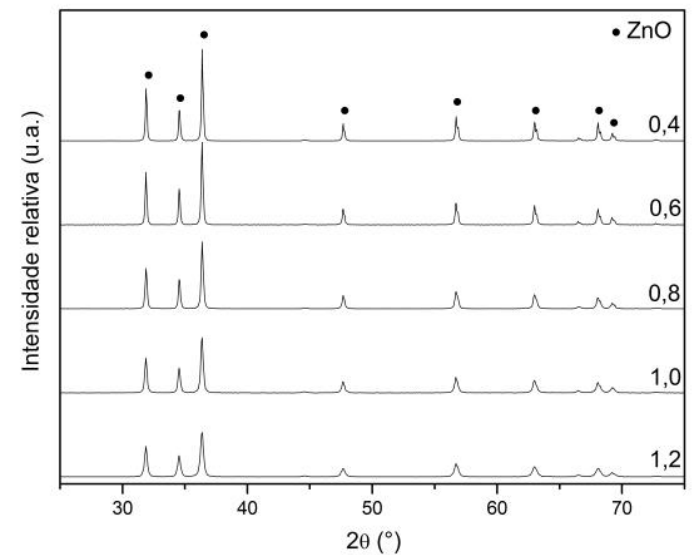

Figura 2-Difratogramas de raios $\mathrm{X}$ dos produtos da síntese de $\mathrm{ZnO}$ por combustão em solução.

A Tabela 2 apresenta os valores para tamanho de cristalito, área superficial e perda de massa para o $\mathrm{ZnO}$ obtido por SCS em função da razão molar utilizada. 
Tabela 2 -Tamanho de cristalito, área superficial e perda de massa para o $\mathrm{ZnO}$ obtido por SCS em função da razão molar combustível/oxidante.

\begin{tabular}{ccccc}
\hline Amostra & $\begin{array}{c}\text { Razão molar } \\
\text { combustível/ } \\
\text { oxidante }\end{array}$ & $\begin{array}{c}\text { Tamanho de } \\
\text { cristalito } \\
(\mathbf{n m})\end{array}$ & $\begin{array}{c}\text { Área } \\
\text { superficial } \\
\left(\mathbf{m}^{2} / \mathbf{g}\right)\end{array}$ & $\begin{array}{c}\text { Perda de } \\
\text { massa (\%) }\end{array}$ \\
\hline U1 & 0,4 & 101 & 4,054 & 1,9 \\
\hline U2 & 0,6 & 87,4 & 4,530 & 2,8 \\
\hline U3 & 0,8 & 60,5 & 4,828 & 11 \\
\hline U4 & 1,0 & 43,9 & 8,082 & 25 \\
\hline U5 & 1,2 & 27,9 & 9,542 & 29 \\
\hline
\end{tabular}

A Figura 3 apresenta o tamanho de cristalito dos óxidos obtidos. Os valores de tamanho de cristalito situam-se entre 27,9 e $101 \mathrm{~nm}$. Observa-se que o tamanho de cristalito das amostras diminuiu à medida que a reação aumentou a quantidade de combustível. A amostra U5, com a maior razão molar combustível/oxidante $(1,26)$, apresentou o menor tamanho de cristalito $(27,9 \mathrm{~nm})$.

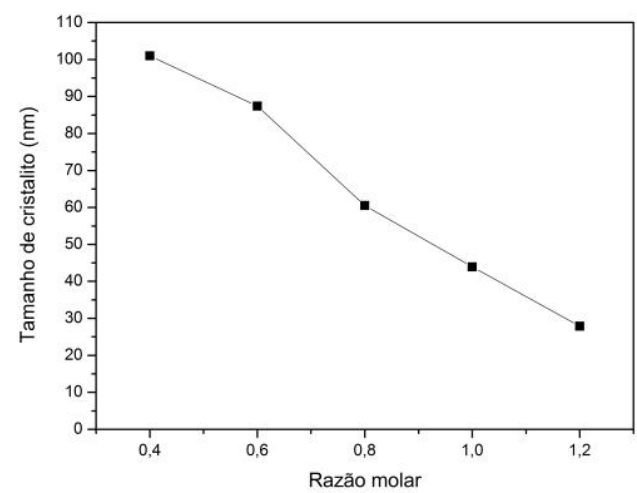

Figura 3 - Tamanho de cristalito dos produtos da síntese de $\mathrm{ZnO}$ por combustão em solução.

A Figura 4 mostra a variação da área superficial dos produtos da síntese de $\mathrm{ZnO}$ por SCS. A área superficial do ZnO é maior para as formulações com excesso de combustível. Este resultado pode ser justificado pela maior quantidade de gás liberada nas reações com maior razão molar combustível/oxidante [20-21].

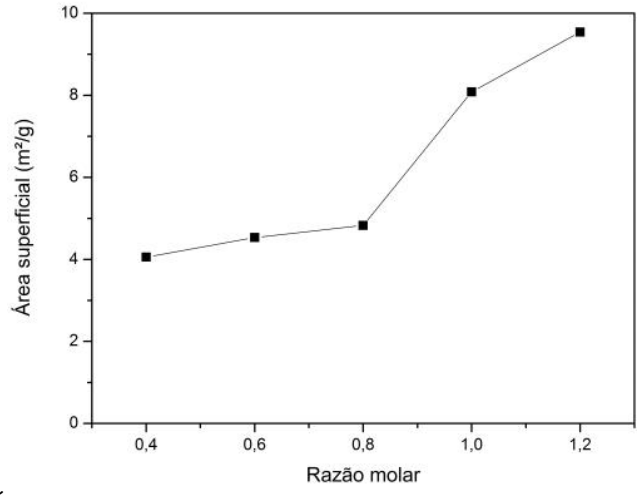

Figura 4 - Área superficial das partículas dos pós-sintetizados via SCS em função da quantidade de combustível.
As Figuras 5a e 5b apresentam as curvas de ATG e ATD dos produtos da síntese de $\mathrm{ZnO}$ por combustão em solução em função da estequiometria dos reagentes na reação. A perda de massa em função da temperatura (Figura 5a) variou significativamente para as formulações estequiométrica (U3) e com excesso de combustível (U4 e U5), cerca de 11\%, 25\% e $29 \%$, respectivamente. Para as amostras deficientes em combustível (U1 e U2) verificou-se perda de massa menor do que $3 \%$. Observa-se na Figura 5a, que a diminuição de massa das amostras ocorre na faixa de temperatura de 50 a $570^{\circ} \mathrm{C}$, possivelmente associada à queima de material orgânico do resíduo de combustão. O platô formado a partir da temperatura de $600^{\circ} \mathrm{C}$ indica a formação de $\mathrm{ZnO}$ nanocristalino como produto da decomposição, confirmado pela análise de DRX. Na curva de ATD (Figura 5b), um pico exotérmico é observado entre 425 e $600^{\circ} \mathrm{C}$, com máximo em torno de $540^{\circ} \mathrm{C}$, para as amostras U3, U4 e U5. Perdas de massa e efeitos térmicos não são observados em temperaturas a cima de $600^{\circ} \mathrm{C}$, indicando que não ocorre decomposição a cima desta temperatura.

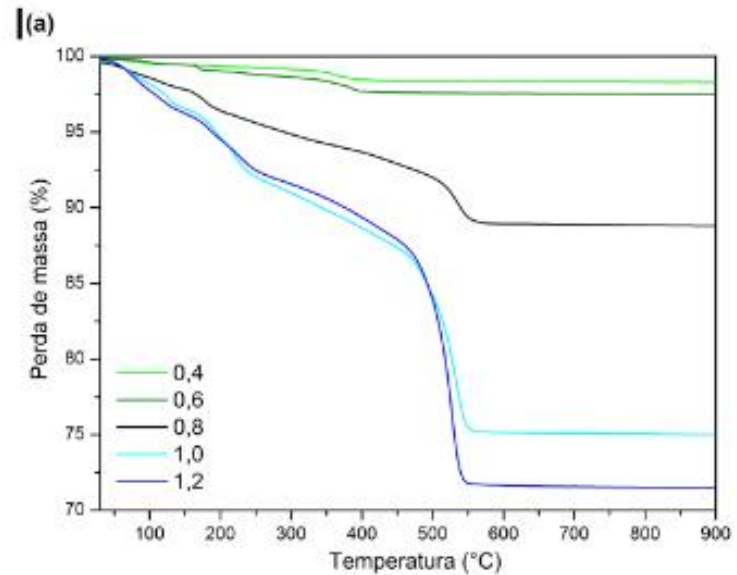

(b)

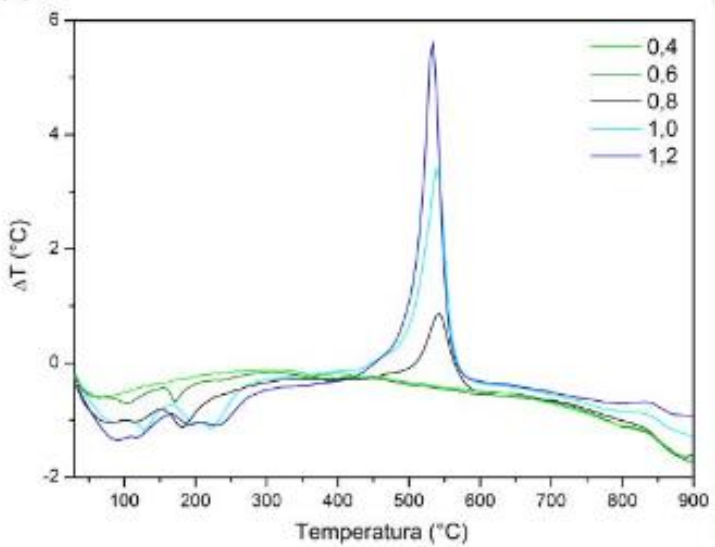

Figura 5 - Curvas de ATG (a) e ATD (b) dos produtos da síntese de ZnO por combustão em solução em função da estequiometria dos reagentes na reação. 
A morfologia dos produtos de síntese foi avaliada por MEV (Figura 6). Os pós-obtidos são constituídos por partículas finas, que possuem grande área superficial e geralmente aglomeram em partículas maiores, a fim de minimizar a superfície total ou a energia interfacial do sistema [22]. Observase a formação de partículas secundárias (agregados) em todas as formulações. A amostra U1 (Figura 6a) apresenta partículas com formatos e tamanhos irregulares. Placas finas com pontas alongadas são observadas para as formulações U2 (Figura 6b) e U3 (Figura 6c), formando uma espécie de conjunto de flores. Nota-se nas amostras U4 (Figura 6d) e U5 (Figura 6e) partículas na forma de placas, sendo que os aglomerados possuem tamanho maior do que as outras formulações.

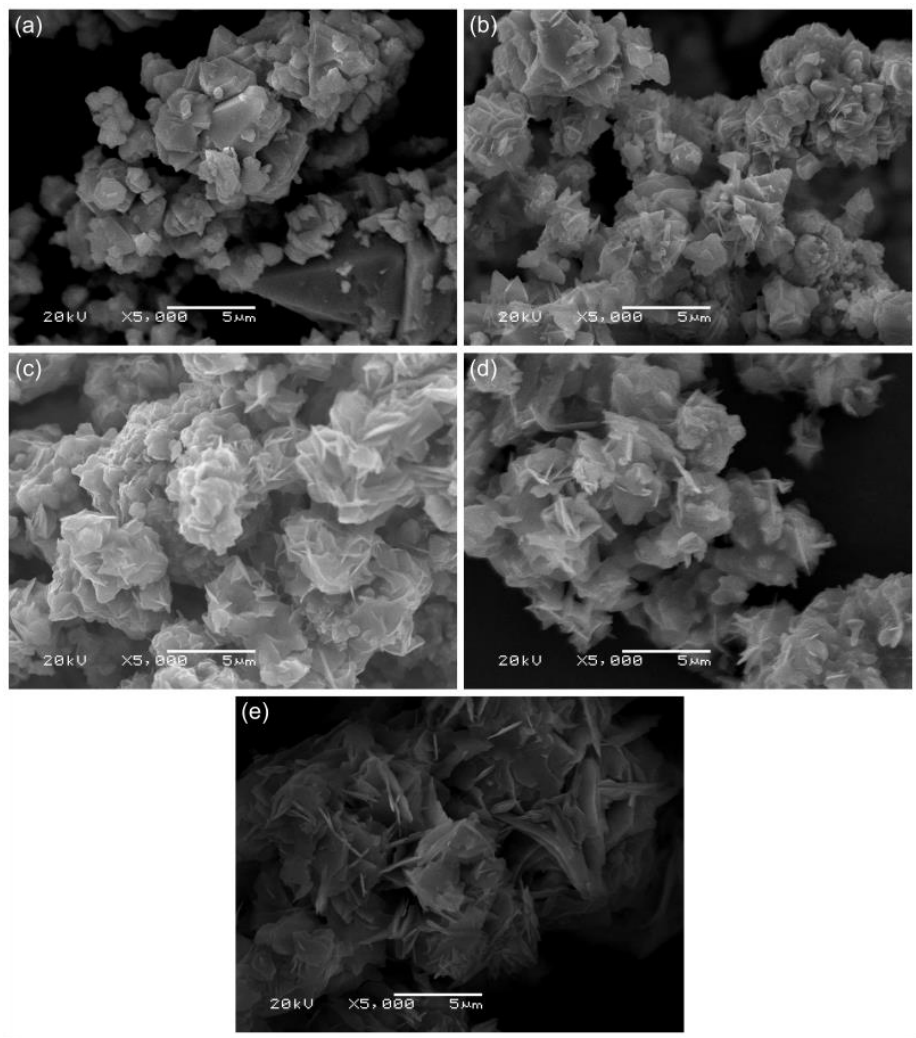

Figura 6 - Imagens de MEV das amostras de ZnO: (a) U1, (b) U2, (c) U3, (d) U4 e (e) U5.

\section{Conclusões}

Este trabalho investigou a influência da razão molar combustível/oxidante na microestrutura do $\mathrm{ZnO}$ obtido por síntese de combustão em solução. De acordo com os resultados apresentados, pode-se concluir o seguinte:

- A cristalinidade dos pós-obtidos variou em função da proporção combustível/oxidante. As formulações com menores razões molares apresentaram picos mais intensos nos difratogramas de raios $\mathrm{X}$;

- Os valores de tamanho de cristalito foram menores para as amostras obtidas com maior quantidade de combustível. O tamanho de cristalito dos $\mathrm{ZnO}$ produzidos variou entre 27,9 e $101 \mathrm{~nm}$;

- A maior quantidade de gases liberada nas reações com excesso de combustível contribuiu para uma maior área superficial do $\mathrm{ZnO}$ sintetizado;

- O ZnO obtido a partir das formulações com excesso de combustível apresentaram maior perda de massa, possivelmente devido à perda de material orgânico remanescente do processo de combustão;

- O formato das partículas do $\mathrm{ZnO}$ sintetizado variou de acordo com a quantidade de ureia utilizada: placas irregulares e placas finas com pontas alongadas à semelhança de flores.

\section{Agradecimentos}

Agradecemos à FAPERGS-PRONEX e o Centro de Microscopia da UFRGS pelo apoio na realização deste trabalho.

\section{INFLUENCE OF FUEL/OXIDANT MOLAR RATIO IN THE MICROSTRUCTURE OF ZINC OXIDE OBTAINED BY COMBUSTION SYNTHESIS IN SOLUTION}

\begin{abstract}
Nanostructured zinc oxide ( $\mathrm{ZnO})$ was obtained by combustion synthesis in solution (CSS) using zinc nitrate as oxidant and urea as fuel. The material was characterized by $\mathrm{X}$ ray diffraction (XRD), Branauer, Emmet and Teller method (BET), thermal gravimetry (TGA) and thermal differential (DTA) analysis, and scanning electron microscopy (SEM). The influence of different fuel/oxidant molar ratios on the microstructure of $\mathrm{ZnO}$ was investigated. The $\mathrm{ZnO}$ powders produced using the highest molar ratio presented the lowest crystallite size and the highest surface area. The SEM images showed irregular plates and thin plates with elongated ends that resemble flowers.
\end{abstract}

Keywords: Nanostructured zinc oxide. Combustion synthesis in solution, Microstructure.

\section{Referências}

[1] CAMPOS, L.C.; Crescimento de nanofios semicondutores de óxido de zinco por deposição química na fase vapor. 2006. 100f. Dissertação (Programa de Pósgraduação em Física - Mestrado) - Universidade Federal de Minas Gerais,Belo Horizonte. 2006 


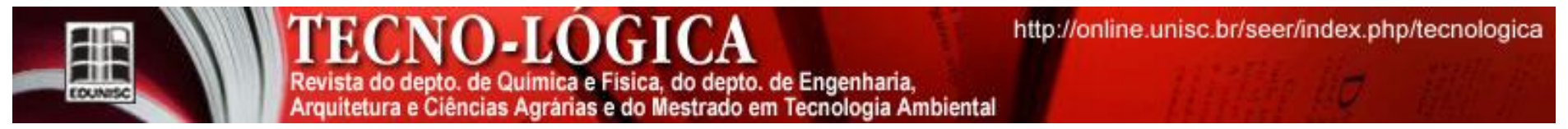

[2] SEKINE, N.; CHOU, C.H.; KWAN, W.L.; YANG, Y.; ZnO nano-ridge structure and its application in inverted polymer solar cell, Organic Electronics, Vol. 10, p. 1473-1477, 2009.

[3] DJURISIC, A.B.; LEUNG, Y.H.; Optical properties of $\mathrm{ZnO}$ nanostructures, Small, Vol. 2, p. 944-961, 2006.

[4] REN, X.; HAN, D.; CHEN,D.; TANG, F.; Large-scale synthesis of hexagonal cone-shaped $\mathrm{ZnO}$ nanoparticles with a simple route and their application to photocatalytic degradation, Materials Research Bulletin, Vol. 42, p. 807-813, 2007.

[5] MATSUOKA, M.; MATSUYAMA, T.; NISHI, T.; Sintering type voltagenonlinear resistor, Japanese patent no. 679748, 1973.

[6] WANG, Z.L.; SONG, J.H.; Piezoelectric nanogenerators based on zinc oxide nanowire arrays, Science, Vol. 312, p. 242-246, 2006.

[7] NOHYNEK, G.J.; LADEMANN, J.; RIBAUD, C.; ROBERTS, M.S.; Nanotechnology, cosmetic and sunscreen safety, Critical Reviews in Toxicology, Vol. 37, p. 251-277, 2007.

[8] WANG, J.; GAO, L.; Hydrothermal synthesis and photoluminescence properties of $\mathrm{ZnO}$ nanowires, Solid State Communications, Vol. 132, p. 269-271, 2004.

[9] PECHINI, M.P.; Method of preparing lead and alkaline: earth, titanates and niobates and coating. Method using the same for a capacitor.U.S. Patent no.3330697, 11 July 1967.

[10] CAO, Y.; LIU, B.; HUANG, R.; XIA, Z.; GE, S.; Flash synthesis of flowerlike $\mathrm{ZnO}$ nanostructures by microwave-induced combustion process, Materials Letters, Vol. 65, p. 160-163, 2011.

[11] SOUSA, V.C.; SEGADÃES, A.M.; MORELLI, M.R.; KIMINAMI, R.H.G.A.; Combustion synthesized $\mathrm{ZnO}$ powders for varistor ceramics, International Journal of Inorganic Materials, Vol. 1, p.235 -241, 1999.

[12] HWANG, C.C.; WU, T.Y.;Synthesis and characterization of nanocrystalline $\mathrm{ZnO}$ powders by a novel combustion synthesis method, Materials Science and Engineering B, Vol. 111,p. 197-206, 2004.

[13] EKAMBARAM, S.;Combustion synthesis and characterization of new class of ZnO-based ceramic pigments, Journal of Alloys and Compounds, Vol. 390, L4-L6, 2005.

[14] SOUSA, V.C.; Varistores de $\mathrm{ZnO}$ obtidos por reação de combustão. Blucher Acadêmico; São Paulo, 2008.177 p.

[15] JAIN, S.R.; Energetics of Propellants, Fuels and Explosives. Propellants, Explosives, Pyrotechnics, Vol. 12, p.188-195, 1987.

[16] GARCIA, A.P.; Síntese de óxido de zinco nanoestruturado por combustão em solução e caracterização de propriedades microestruturais e atividade fotocatalítica. 2011. 60 f. Dissertação (Programa de Pós-graduação em Engenharia de Minas, Metalúrgica e de Materiais - Mestrado) - Universidade Federal do Rio Grande do Sul, Porto Alegre.2011.

[17] ONISEI, S.; BADILITA, V.; STOICIU, F.; VELEA, T.; PREDICA, V.; LUPU, C.;BUTU, M.; MOLDOVAN, P.; Mechanochemical Activation of Copper Concentrate and the Effect on Oxidation of Metal Sulphides, Revista de Chimie,Vol. 63, p. 591-597, 2012.
[18] BRANAUER, S.; EMMETT, P.H.; TELLER, E.; Adsorption of gases in multimolecular layers, Journal American Chemical Society, Vol. 60, p. 309-319, 1938 .

[19] WEBB, P. A.; ORR, C.; Analytical methods in fine particle technology. Micromeritics Instrument Corporation; Norcross, 1997. 301p.

[20] HWANG, C.C.; WU, T.Y.; Combustion synthesis of nanocrystalline ZnO powders using zinc nitrate and glycine as reactants-influence of reactant composition, Journal of Materials Science, Vol. 39, p. 6111-6115, 2004.

[21] TONIOLO, Juliano Cantarelli. Influência da razão combustível - oxidante nas características de óxidos nanoestruturados sintetizados por combustão em solução. 2009. 156 f. Tese (Programa de Pós-Graduação em Engenharia de Minas, Metalúrgica e de Materiais - Doutorado) - Universidade Federal do Rio Grande do Sul, Porto Alegre.2009.

[22] EDELSTEIN, A.S.; CAMARATA, R.C.; Nanomaterials: synthesis, properties and applications. Physics Publishing; Londres, 1996. 